\title{
Purinergic signalling in the immune system. A brief update
}

\author{
Francesco Di Virgilio
}

Received: 10 October 2006 / Accepted: 10 October 2006 / Published online: 6 February 2007

(C) Springer Science + Business Media B.V. 2007

Signalling by extracellular ATP has become a focus of hot interest in virtually any field in biomedical sciences, from developmental biology to neurophysiology, from kidney diseases to ophthalmology and from osteoporosis to cancer [1]. One of the fields that has lagged behind this, at times tumultuous, development has been immunology. Although reports from many laboratories have provided ample demonstration that extracellular ATP and P2 receptors are key players in the activation phases of the immune response, the hypothesis that the purinergic system might be a relevant pathway in host defense is surrounded by benign skepticism in the immunological community. However, despite this difficult acceptance, interest is slowly rising even among immunologists, as witnessed by the steady increase in the number of papers reporting on the effect of purinergic agonists in many different immune-mediated responses.

Although the molecular details are often lacking a wealth of observations emphasize the central role of extracellular nucleotides in chemotaxis, cytokine secretion, cell fusion, surface antigen shedding, intracellular pathogen killing and inflammatory pain (see [2] for a recent review). Also adenosine is enjoying a new life in immunology as novel data support a crucial role of $\mathrm{P} 1$ receptors in the coordinated tissue response in acute and chronic inflammation under normoxic and hypoxic conditions [3]. Anti-inflammatory effects of adenosine seem to be mainly mediated via the A2A receptor, while extracellular ATP as an immunomodulatory agent acts both at $\mathrm{P} 2 \mathrm{Y}$ and $\mathrm{P} 2 \mathrm{X}$ receptors.

F. Di Virgilio $(\square)$

Department of Experimental and Diagnostic Medicine, Section of General Pathology, and Interdisciplinary Center for the Study of Inflammation, University of Ferrara,

Via Borsari 46,

44100 Ferrara, Italy

e-mail: fdv@unife.it
Among the $\mathrm{P} 2 \mathrm{X}$ receptors, the $\mathrm{P} 2 \mathrm{X}_{7}$ subtype is gaining an increasingly relevant role in the overall economy of cell response to bacterial or host-derived noxious agents as we learn more about the cellular integration of proinflammatory signals by phagocyte sensors (whether located in the plasma membrane or in the cytoplasm) and effector pathways $[4,5]$. In fact, accumulating evidence shows that the maturation and release of proinflammatory cytokines of the interleukin (IL)-1 family are mediated by a caspase-1activating platform referred to as "inflammasome" which is activated by pathogen- or host-derived factors, such as muramyl dipeptide, uric acid crystals and calcium pyrophosphate [6]. These molecules directly interact with a central component of the inflammasome known a NALP3/ CIAS1/cryopyrin. Recent data show that extracellular ATP via the $\mathrm{P}_{2} \mathrm{X}_{7}$ receptor is one of the most potent physiological stimuli for the inflammasome in a NALP3-dependent fashion [7]. The very recent data implicating ATP in the activation of the inflammasome nicely complement previous reports showing a crucial role of this nucleotide in the maturation and release of key cytokines such as IL-1 $\alpha$, IL$1 \beta$ and IL-18 [4].

Furthermore, converging data from several laboratories clearly show that chronic exposure of dendritic cells to low ATP doses, as it may happen at sites of enduring low level tissue damage by agents with low pathogenicity, has a profound effect on dendritic cell differentiation, favouring the development of a Th2-skewing dendritic cell phenotype [8]. Finally, the demonstration that adenosine A2A receptors have a non-redundant inhibitory role in preventing inflammatory and immune cell activation nicely completes the scenario illustrating the homeostatic function of purinergic signalling in immunity [9]. Altogether, these exciting observations bring purinergic signalling to the heart of immunity and inflammation. 
Any stressed or injured cell releases ATP via lytic or, most likely, non-lytic pathways, thus generating a very early and sensitive sign of cellular distress ("danger signal"). ATP release quickly alerts the immune system of an impending danger due to exogenous or endogenous causes. In this view, purinergic receptors may function as sensors of danger endowed with the ability to mould the immune response according to the source, amount and duration of danger signal/intracellular nucleotide release. An acute, massive discharge of ATP into the extracellular space will act as a potent co-stimulus for release of proinflammatory cytokines of the IL-1 family and might even further enhance tissue damage by exerting a direct cytotoxic effect. On the contrary, a smaller but long lasting release will have a less dramatic and more subtle effect by stimulating phagocyte chemotaxis, upregulating chemokine receptors and driving dendritic cell differentiation. As it is typical of any homeostatic system, the activating arm will also turn on a deactivating loop, represented in this case by the accumulation of adenosine, which powerfully depresses immune cell functions. Needless to say, the potential applications to the therapy of inflammatory diseases are countless (Fig. 1).

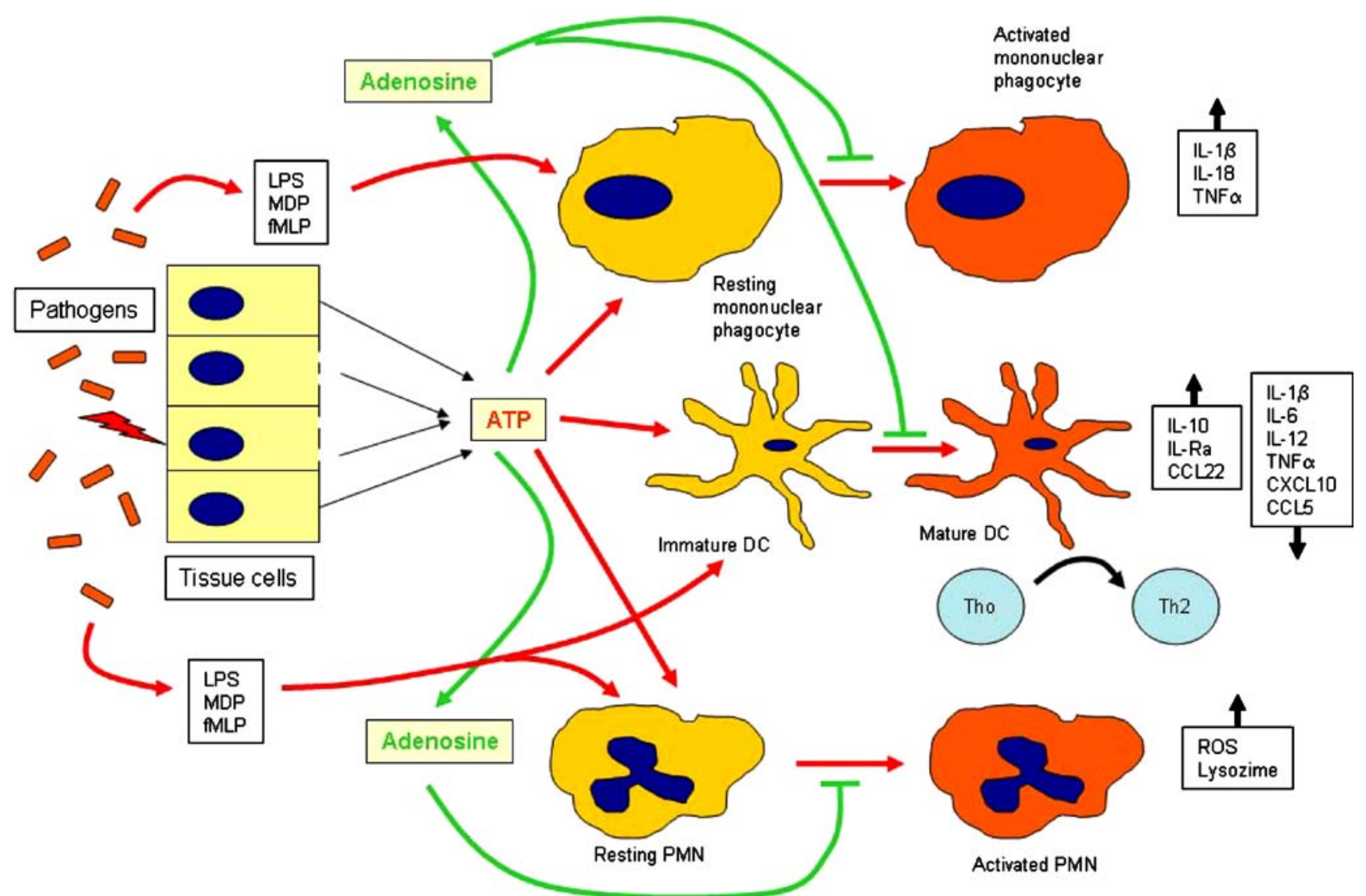

Fig. 1 Purinergic signalling in the activation/deactivation of the innate immune response. Pathogens release factors that activate innate immunity either directly [e.g. lipopolysaccharide (LPS), muramyl dipeptide $(M D P)$, formyl-methionyl-leucyl-phenylalanine $(f M L P)]$ or indirectly by causing injury or distress of host cells (e.g. ATP). Bacterial factors, which are also known as "exogenous danger signals", recruit and stimulate tissue macrophages, dendritic cells $(D C)$ and polymorphonuclear leukocytes $(P M N)$. Activated inflammatory cells are shown in red. Extracellular ATP, which accumulates at sites of inflammation and may be considered an "endogenous danger signal", modulates the activity of pathogen-derived factors in different ways (red arrows). For example, ATP can exert a synergistic, proinflammatory effect, by increasing cytokine release (e.g. $I L-1 \beta, I L$ $18, T N F \alpha$ ) from endotoxin-primed mononuclear phagocytes, or by stimulating secretion of bactericidal factors (e.g. lysozyme) and synthesis of reactive oxygen species (ROS) by PMN. These responses are mediated via the $\mathrm{P}_{2} \mathrm{X}_{7}$ as well as other $\mathrm{P} 2$ receptors. On the $\mathrm{DC}$, the effect of extracellular ATP in conjunction with bacterial-derived factors is more complex as while high concentrations cause a strong proinflammatory activation, chronic exposure to low concentrations drive DC maturation toward a phenotype favouring the development of a Th2 response. The DC modulatory effects of ATP are mainly mediated via the $\mathrm{P}_{2} \mathrm{Y}_{11}$ receptor. Thus, LPS-maturated and ATPstimulated DC show a high release of anti-inflammatory cytokines, such as IL-10 and IL-Ra, and of chemokines driving a preferential recruitment of Th2 lymphocytes (such as CCL22). Vice versa, LPSmaturated and ATP-stimulated DC downmodulate secretion of IL-1 $\beta$, IL-6, IL-12, TNF $\alpha$, and of chemokines, such as CCL5 and CXCL10, that preferentially recruit Th1 cells. In the extracellular milieu ATP is degraded by plasma membrane ecto-ATPases (such as CD39) and generates adenosine, which by acting at $\mathrm{A} 2 \mathrm{~A}$ receptors has a profound downmodulatory role on inflammation and immunity as a whole (green lines) 


\section{References}

1. Burnstock G (2006) Pathophysiology and therapeutic potential of purinergic signaling. Pharmacol Rev 58:58-86

2. Di Virgilio F, Baricordi OR, Romagnoli R, Baraldi PG (2005) Leukocyte P2 receptors: a novel target for anti-inflammatory and anti-tumor therapy. Curr Drug Targets Cardiovasc Haematol Disord 5: 85-99

3. Sitkovsky M, Lukashev D (2005) Regulation of immune cells by local-tissue oxygen tension: HIF $1 \alpha$ and adenosine receptors. Nat Rev Immunol 5:712-721

4. Ferrari D, Pizzirani C, Adinolfi E et al (2006) The $\mathrm{P} 2 \mathrm{X}_{7}$ receptor: a key player in IL-1 processing and release. J Immunol 176:3877-3883
5. Ogura Y, Sutterwala FS, Flavell RA (2006) The inflammasome: first line of the immune response to cell stress. Cell 126:659-662

6. Martinon F, Petrilli V, Mayor A, Tardivel A, Tschopp J (2006) Gout-associated uric acid crystals activate the NALP3 inflammasome. Nature 440:237-241

7. Sutterwala FS, Ogura Y, Szczepanik M et al (2006) Critical role for NALP3/CIAS1/Cryopyrin in innate and adaptive immunity through its regulation of caspase-1. Immunity 24:317-327

8. la Sala A, Ferrari D, Di Virgilio F et al (2003) Alerting and tuning the immune response by extracellular nucleotides. J Leukoc Biol 73:339-343

9. Sitkovsky MV, Ohta A (2005) The 'danger' sensors that STOP the immune response: the A2 adenosine receptors? Trends Immunol 26:299-304 